\title{
Numerical model of planar heterojunction organic solar cells
}

\author{
MA ChaoZhu ${ }^{1}$, PENG YingQuan ${ }^{1,2^{*}}$, WANG RunSheng ${ }^{1}$, LI RongHua ${ }^{1}$, XIE HongWei ${ }^{1}$, \\ WANG Ying ${ }^{1}$, XIE JiPeng ${ }^{1,3} \&$ YANG Ting ${ }^{1}$ \\ ${ }^{1}$ Institute of Microelectronics of the School of Physical Science and Technology, Lanzhou University, Lanzhou 730000, China; \\ ${ }^{2}$ Key Laboratory for Magnetism and Magnetic Materials of the Ministry of Education, Lanzhou University, Lanzhou 730000, China; \\ ${ }^{3}$ Department of Basic, Airforce Communication Sergeant Accademy, Dalian 116600, China
}

Received March 20, 2010; accepted December 15, 2010

\begin{abstract}
We present a numerical study of the effects of the energy barrier between the lowest unoccupied molecular orbital of the acceptor layer and the cathode, the thicknesses of the donor layer and acceptor layer on the distributions of carrier density, the electric fields and the electric potentials of organic planar heterojunction solar cells. We obtained the quantitative dependencies of the distribution of carrier density, electric fields and the electric potentials on these quantities. The results provide a theoretical foundation for the experimental study of open-circuit organic planar heterojunction solar cells.
\end{abstract}

energy barrier, donor thickness, acceptor thickness, carrier density, heterojunction solar cells

Citation: $\quad$ Ma C Z, Peng Y Q, Wang R S, et al. Numerical model of planar heterojunction organic solar cells. Chinese Sci Bull, 2011, 56: 2050-2054, doi: 10.1007/ s11434-011-4376-4

In recent years, organic solar cells have attracted a great deal of attention. They exhibit several advantages over inorganic solar cells, such as fabrication at low temperatures, bendable and low cost. Since the first report by Tang [1], organic solar cells have been widely investigated and many different structures have been developed. Theoretical studies on single layer organic solar cells [2,3] and bulk-heterojunction organic solar cells $[4,5]$ have been presented. Several models have been developed for planar heterojunction organic solar cells based on equivalent circuit models [6], experimental work [7] or semi-analytical approaches [8]. Despite growing research efforts, the fundamental processes governing the operation of planar heterojunction organic solar cells are still poorly understood. These models do not account for internal physical mechanisms such as the carrier distribution and electric field.

In this work, we derive a numerical model for distribution of carriers, fields and potentials based on the diffusion and drift of carriers in both donor and acceptor materials.

*Corresponding author (email: yqpeng@1zu.edu.cn)
Then we investigate behavior of the energy barrier between the lowest unoccupied molecular orbitals (LUMO) of the acceptor layer and the cathode, the influence of the thicknesses of the donor layer and acceptor layer on the distribution of carriers and the distribution of the electric fields and the electric potentials in organic planar heterojunction solar cells. The results provide a theoretical foundation for the experimental study of open-circuit organic planar heterojunction solar cells.

\section{Theoretical model}

When the planar heterojunction organic solar cell is illuminated, a large number of excitons (the bound electron-hole pairs) are created in the organic layer of the solar cell. These excitons dissociate into free electrons and holes after they diffuse across the interface between the donor and acceptor materials. These free electrons and holes are transported to their respective electrodes under the influence of the intrinsic electrical field, which can be seen in Figure 1. 
Experimental results show that the trap effect can be neglected because of its weak influence on the carrier transport in organic solar cells [9]. Therefore, the spatial distribution of the potential $\psi(x)$, the electron density $n(x)$ and the hole density $p(x)$ at position $x$ are related through the position equation:

$$
\frac{\partial^{2}}{\partial x^{2}} \psi(x)=\frac{q}{\varepsilon_{r} \varepsilon_{0}}[n(x)-p(x)],
$$

where $\varepsilon_{r}$ is the relative permittivity, $\varepsilon_{0}$ is the vacuum permittivity, $q$ is the elementary charge. If we assume the device to be in steady state and account for the donor and acceptor materials, eq. (1) can be written as

$$
\frac{\mathrm{d} F}{\mathrm{~d} x}= \begin{cases}\frac{q}{\varepsilon_{r} \varepsilon_{0}} p(x) & 0 \leqslant x<d_{D}, \\ -\frac{q}{\varepsilon_{r} \varepsilon_{0}} n(x) & d_{\mathrm{D}} \leqslant x \leqslant d_{\mathrm{D}}+d_{\mathrm{A}},\end{cases}
$$

where $d_{\mathrm{D}}$ and $d_{\mathrm{A}}$ are the thicknesses of the donor layer and acceptor layer, respectively.

If we consider the one dimensional case, the donor layer consists of hole-conducting donor material and the acceptor layer consists of electron-conducting donor material. As a result, the total current in the donor and acceptor layers can be written as

$$
\begin{aligned}
& j_{p}=q\left(\mu_{p} p F-D_{p} \frac{\mathrm{d} p}{\mathrm{~d} x}\right) \\
& j_{n}=q\left(\mu_{n} n F+D_{n} \frac{\mathrm{d} n}{\mathrm{~d} x}\right),
\end{aligned}
$$

where $F$ is the electric field strength, $D_{n(p)}$ is the electron (hole) diffusion coefficient and $D_{n(p)}$ is the electron (hole) mobility. These quantities are assumed to obey the Einstein relation:

$$
\frac{D_{n(p)}}{\mu_{n(p)}}=\frac{k_{B} T}{q},
$$

were $k_{B}$ is the Boltzmann's constant and $T$ is the temperature. We then substitute eq. (4) into eq. 3(a) and 3(b) and obtain:

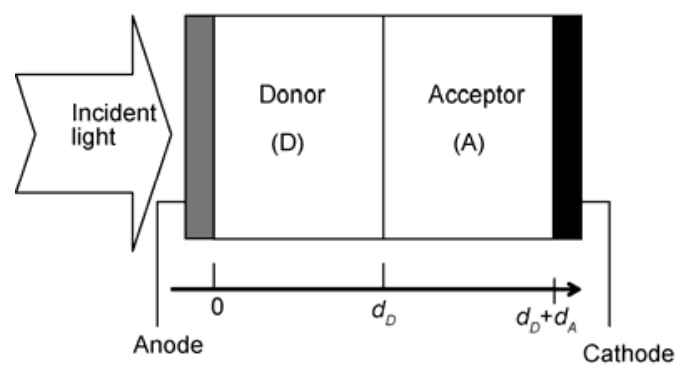

Figure 1 Structure of planar heterojunction organic solar cells.

$$
\begin{aligned}
& j_{p}=q \mu_{p}\left(p F-\frac{k_{B} T}{q} \frac{\mathrm{d} p}{\mathrm{~d} x}\right), \\
& j_{n}=q \mu_{n}\left(n F+\frac{k_{B} T}{q} \frac{\mathrm{d} n}{\mathrm{~d} x}\right) .
\end{aligned}
$$

For an open-circuit configuration, $j_{p}=0, j_{n}=0$ and eqs. 5(a) and 5(b) become

$$
\begin{aligned}
& q \mu_{p}\left(p F-\frac{k_{B} T}{q} \frac{\mathrm{d} p}{\mathrm{~d} x}\right)=0, \\
& q \mu_{n}\left(n F+\frac{k_{B} T}{q} \frac{\mathrm{d} n}{\mathrm{~d} x}\right)=0 .
\end{aligned}
$$

The hole density, $p_{a}$, at $x=0$ can be written as

$$
p_{a}=N_{\mathrm{D}} \exp \left(-\frac{\Delta \Phi_{a}}{k_{B} T}\right),
$$

were $N_{D}$ is the effective density of states of the donor layer and $\Delta \Phi_{a}$ is the energy barrier between the highest occupied molecular orbital energy level of the donor layer and the Fermi level of the anode. The electron density, $n_{c}$, at $x=d_{\mathrm{D}}+d_{\mathrm{A}}$ can be written as:

$$
n_{c}=N_{\mathrm{A}} \exp \left(-\frac{\Delta \Phi_{c}}{k_{B} T}\right),
$$

where $N_{\mathrm{A}}$ is the effective density of states of the acceptor layer and $\Delta \Phi_{c}$ is the energy barrier between the LUMO energy of the acceptor layer and the Fermi energy of the cathode.

The hole density and the electron density at the interface $x=d_{\mathrm{D}}$ are satisfied by the following condition:

$$
\left.\frac{\mathrm{d} p}{\mathrm{~d} x}\right|_{x=d_{\mathrm{D}}}=\left.\frac{\mathrm{d} n}{\mathrm{~d} x}\right|_{x=d_{\mathrm{D}}} .
$$

To study the interplay among the distributions, fields and parameters, we present a numerical approach to solving eqs. (1)-(9).

\section{Results and discussion}

Correlating the carrier density with incident light intensity is difficult. Therefore, the plots used in this paper give the carrier density, $p_{i, s}$, at the interface on the $x$ axis instead of the incident light intensity.

\subsection{Energy barrier effects}

Figure 2 shows the effect of $\Delta \Phi_{c}$ on the calculated distribution of carrier densities, electric field and electric potential for a given $\Delta \Phi_{a}$ and the Fermi level of the anode. It can be 

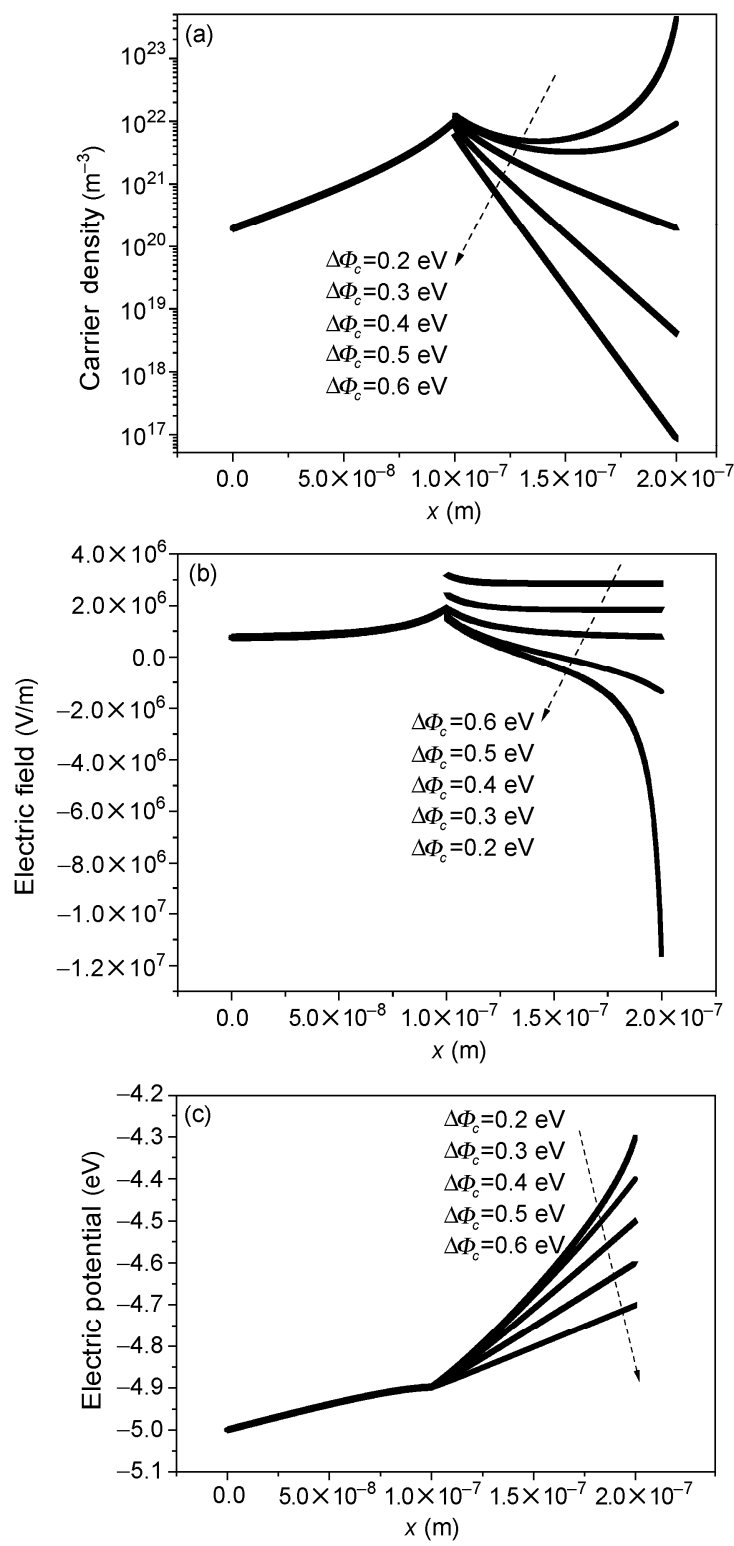

Figure 2 Effect of $\Delta \Phi_{c}$ on the distribution of carriers, the electric field and the electric potential for $\Delta \Phi_{a}=0.4 \mathrm{eV}, N_{\mathrm{D}}=N_{\mathrm{A}}=1.0 \times 10^{27} \mathrm{~m}^{-3}, p_{i, s}=$ $1.0 \times 10^{22} \mathrm{~m}^{-3}, \varepsilon_{\mathrm{D}}=\varepsilon_{A}=3.0, d_{\mathrm{D}}=d_{\mathrm{A}}=100 \mathrm{~nm}, T=300 \mathrm{~K}$, and $W_{a}=-5.0$ $\mathrm{eV}, \mathrm{HOMO}_{\mathrm{D}}=-5.4 \mathrm{eV}, \mathrm{HOMO}_{\mathrm{A}}=-5.7 \mathrm{eV}, \mathrm{LUMO}_{\mathrm{D}}=-3.9 \mathrm{eV}, \mathrm{LUMO}_{\mathrm{A}}$ $=-4.1 \mathrm{eV}$. (a) Calculated distribution of carriers; (b) calculated distribution of the electric field; (c) calculated distribution of the electric potential.

seen from Figure 2(a), 2(b) and 2(c) that the hole density, the electric field and the electric potential in the donor layer vary little with $\Delta \Phi_{c}$. However, there is an obvious change in the electron density, the electric field and the electric potential in the acceptor layer. For a given $\Delta \Phi_{a}$, the electron density and the electric potential vary inversely with $\Delta \Phi_{c}$. This can be explained by the fact that the electron density in the acceptor layer near the cathode decreases with increasing $\Delta \Phi_{c}$, according to eq. (8). This leads to decreasing electron density at the interface between the donor layer and the acceptor layer for the same gradient of the electron density distribution, according to eq. (9). This trend leads to decreasing electron density in the acceptor layer for $x$ from $d_{\mathrm{D}}$ to $d_{\mathrm{D}}+d_{\mathrm{A}}$. The decreasing electron density causes an increase of the electric field in the acceptor layer for a given $p_{i, s}$ and $\Delta \Phi_{a}\left(\Delta \Phi_{a}=0.4 \mathrm{eV}\right)$, according to the eq. (2). The electric potential has a trend opposite that of the electric field, which is shown in Figure 2(c).

\subsection{Donor layer thickness}

Figure 3 shows the effect of the donor layer thickness on the distribution of carriers, the electric field and the electric potential for a given acceptor layer thickness. It can be seen from Figure 3(a) that when the donor layer thickness is increased, the distribution of holes in the donor layer broadens and flattens. As a result, the gradient of the hole density distribution at the interface $x=d_{\mathrm{D}}$ decreases. This leads the electron density at the interface between the donor layer and the acceptor layer to decrease. Therefore, the electric field at this interface will follow the same trend. This can be seen in Figure 3(b). According to eq. (2), this leads to increasing electric potential with increasing donor layer thickness, which can be seen in Figure 3(c).

\subsection{Acceptor layer thickness}

Figure 4 shows the effect of the acceptor layer thickness on the distribution of carriers, the electric field and the electric potential for a given donor layer thickness. From these figures it can been seen that the distribution of hole density, the electric field and the electric potential in the donor layer are almost constant even when there are significant variations in electron density, the electric field distribution and the electric potential distribution in the acceptor layer. It can be seen in Figure 4(a) that the distribution of electrons in the acceptor layer broadens when the thickness of the acceptor is increased, for a given donor layer thickness. At the interface between the donor layer and the acceptor layer, the gradient of the hole density distribution is constant. Therefore, the gradient of the electron density distributions at these interfaces must also be almost constant. This leads to the broadening of the electron density distribution. According to eq. (2), this results in a similar trend for the electric field distribution, which can be seen in Figure 4(b) and 4(c). This leads to the conclusion that the electric potential distribution in planar heterojunction organic solar cells varies positively with the electron density in the acceptor layer.

\section{Conclusion}

In this paper, we numerically studied the effects of $\Delta \Phi_{c}$, $\Delta \Phi_{a}$, and the thicknesses of the donor and acceptor layers on three distributions: the carrier density distribution, the electric field distribution and the electric potential distribu- 

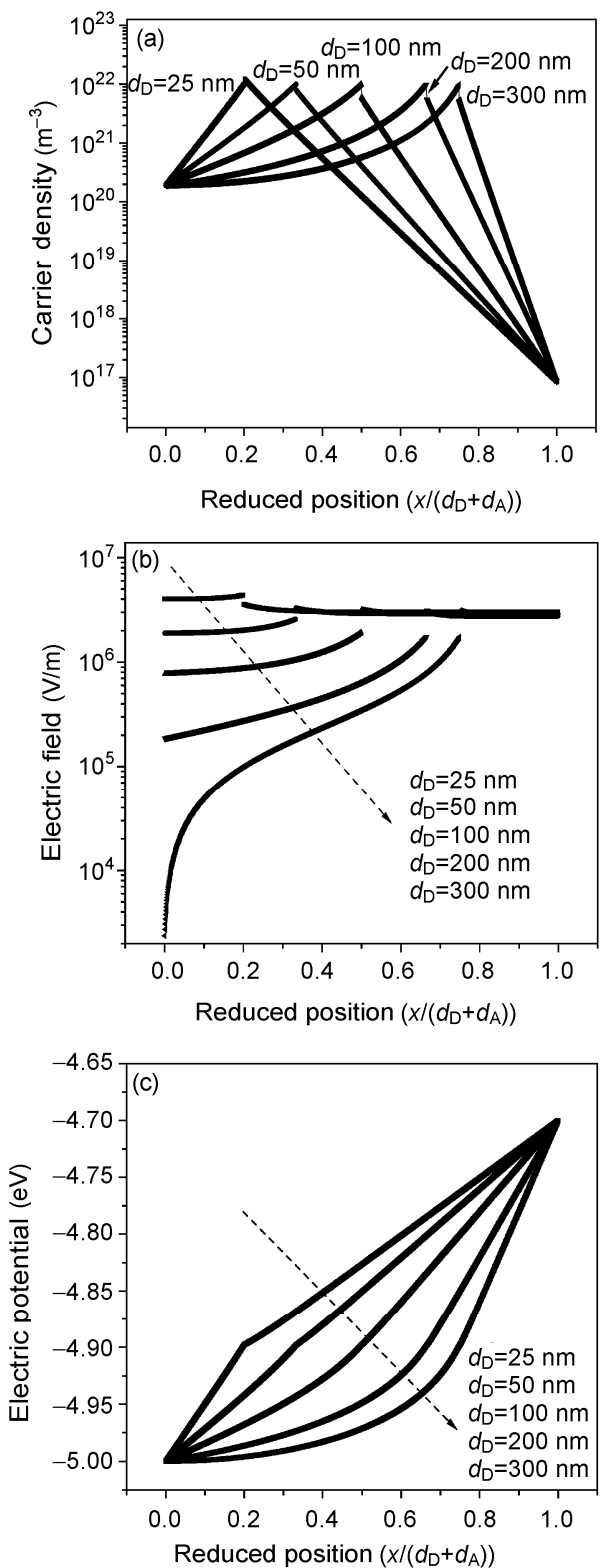

Figure 3 Effect of the donor layer thickness on the carrier distribution, electric field and electric potential, with $N_{\mathrm{D}}=N_{\mathrm{A}}=1.0 \times 10^{27} \mathrm{~m}^{-3}, p_{i, s}=$ $1.0 \times 10^{22} \mathrm{~m}^{-3}, \varepsilon_{\mathrm{D}}=\varepsilon_{\mathrm{A}}=3.0, d_{\mathrm{A}}=100 \mathrm{~nm}, T=300 \mathrm{~K}, W_{\mathrm{a}}=-5.0 \mathrm{eV}, W_{\mathrm{c}}=-4.7$ $\mathrm{eV}, \mathrm{HOMO}_{\mathrm{D}}=-5.4 \mathrm{eV}, \mathrm{HOMO}_{\mathrm{A}}=-5.7 \mathrm{eV}, \mathrm{LUMO}_{\mathrm{D}}=-3.9 \mathrm{eV}, \mathrm{LUMO}_{\mathrm{A}}=$ $-4.1 \mathrm{eV}, \Delta \Phi_{a}=0.4 \mathrm{eV}$ and $\Delta \Phi_{c}=0.6 \mathrm{eV}$. (a) Calculated distribution of carriers; (b) calculated distribution of the electric field; (c) calculated distribution of the electric potential.

tion of organic planar heterojunction solar cells. The results indicate that for a given $\Delta \Phi_{a}$, the hole density, the electric field and the electric potential in the donor layer vary little. The electron density in the acceptor layer decreases with increasing $\Delta \Phi_{c}$. However, the electric field in the acceptor layer increases. For a given acceptor layer thickness, the electron density at the interface between the donor layer and the acceptor layer decreases with increasing donor layer thickness. This results in the broadening and flattening of the electron density distribution in the acceptor layer. The
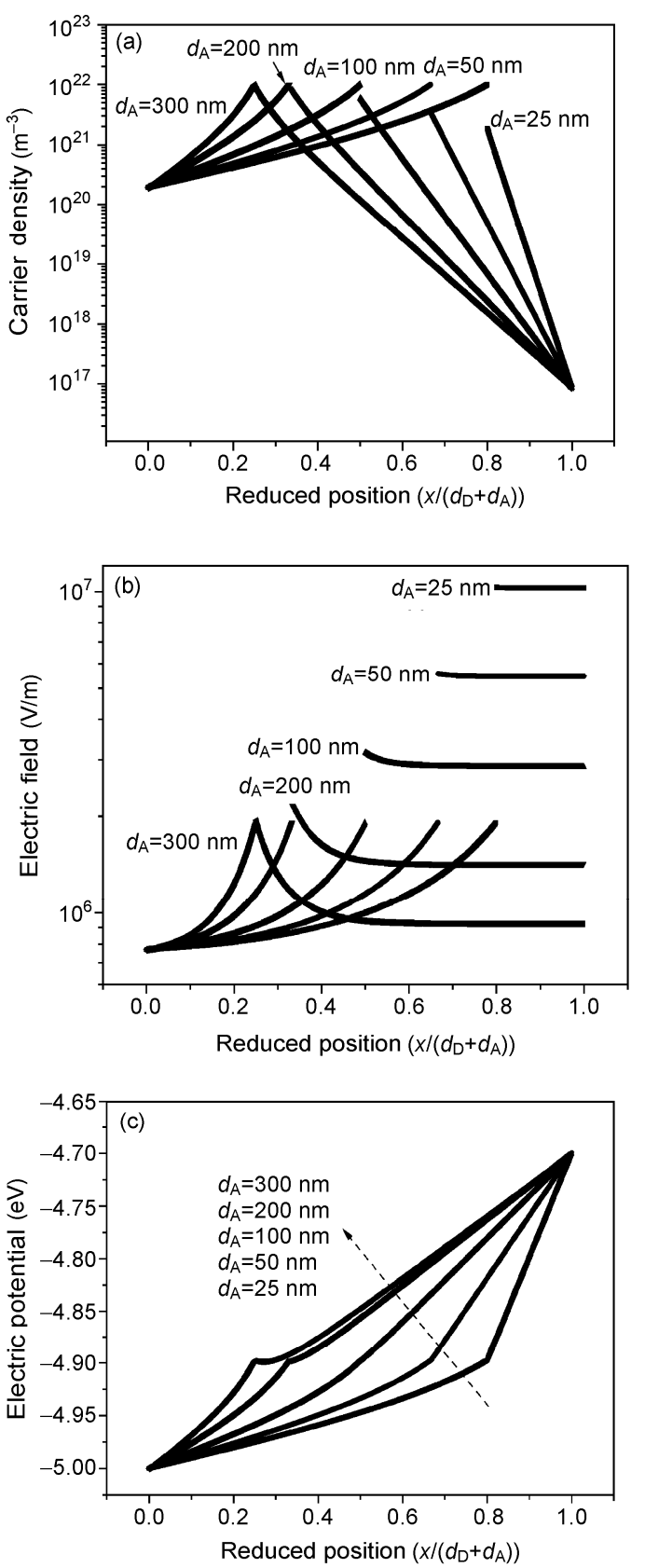

Figure 4 Effect of the acceptor layer thickness on the carrier distribution, electric field and electric potential, where $N_{\mathrm{D}}=N_{\mathrm{A}}=1.0 \times 10^{27} \mathrm{~m}^{-3}, p_{i, s}=$ $1.0 \times 10^{22} \mathrm{~m}^{-3}, \varepsilon_{\mathrm{D}}=\varepsilon_{\mathrm{A}}=3.0, d_{\mathrm{D}}=100 \mathrm{~nm}, T=300 \mathrm{~K}, W_{a}=-5.0 \mathrm{eV}, W_{c}=-4.7$ $\mathrm{eV}, \mathrm{HOMO}_{\mathrm{D}}=-5.4 \mathrm{eV}, \mathrm{HOMO}_{\mathrm{A}}=-5.7 \mathrm{eV}, \mathrm{LUMO}_{\mathrm{D}}=-3.9 \mathrm{eV}, \mathrm{LUMO}_{\mathrm{A}}=$ $-4.1 \mathrm{eV}, \Delta \Phi_{a}=0.4 \mathrm{eV}$ and $\Delta \Phi_{c}=0.6 \mathrm{eV}$. (a) Calculated distribution of carriers; (b) calculated distribution of the electric field; (c) calculated distribution of the electric potential.

electric field in the acceptor layer follows the same trend. For a given donor layer thickness, the gradient of the electron density at the interface between the donor layer and the acceptor layer is nearly constant. This leads to the broadening of the distributions of the electron density, electric field, and electric potential in the acceptor layer of a planar heterojunction organic solar cell. 
This work was supported by the National Natural Science Foundation of China (10974074) and the Natural Science Foundation of Gansu Province (0803RJZA104)

1 Tang C W. Two-layer organic photovoltaic cell. Appl Phys Lett, 1986, 48: $183-185$

2 Guo Z J, Xing H W, Peng Y Q, et al. Effects of carrier mobility, energy gap, and excitation size on the performance of single layer organic solar cells. Optoelectron Lett, 2008, 4: 0410-0414

3 Li R H, Meng W M, Peng Y Q, et al. Investigation on the effect of cathode work function and exciton generation rate on the open-circuit voltage of single layer organic solar cells with Schottky contact. Acta Phys Sin, 2010, 59: 2126-2130

4 Xing H W, Peng Y Q, Yang Q S, et al. Simulation of polymer- fullerene bulk heterojunction solar cell. Acta Phys Sin, 2008, 57: 7374-7379

5 Sasa L J, Olle I. Modeling electrical transport in blend heterojunction organic solar cells. J Appl Phys, 2005, 97: 124901

6 Mazhari B. An improved solar cell circuit model for organic solar cells. Sol Energy Mater Sol Cells, 2006, 90: 1021-1033

7 Scharber M C, Mühlbacher D, Koppe M, et al. Design rules for donors in bulk-heterojunction solar cells-towards 10\% energy-conversion efficiency. Adv Mater, 2006, 18: 789-794

8 Barker J A, Ramsdale C M, Greenham N C, Modeling the currentvoltage characteristics of bilayer polymer photovoltaic devices. Phys Rev B, 2003, 67: 075205

9 Koster L J A, Smits E C P, Mihailetchi V D, et al. Device model for the operation of polymer/fullerene bulk heterojunction solar cells. Phys Rev B, 2005, 72: 085205

Open Access This article is distributed under the terms of the Creative Commons Attribution License which permits any use, distribution, and reproduction in any medium, provided the original author(s) and source are credited. 